\title{
Respiratory epithelial ion transport in patients with disseminated bronchiectasis
}

\author{
I. Danner*, ${ }^{,+}$P. Boisseau**, E. Chailleux*, D. Escande ${ }^{*+}$
}

Respiratory epithelial ion transport in patients with disseminated bronchiectasis. I. Danner, P. Boisseau, E. Chailleux, D. Escande. (C)ERS Journals Ltd 1999.

ABSTRACT: The nosological limits between disseminated bronchiectasis and cystic fibrosis (CF) remain unclear. In patients with isolated congenital bilateral absence of the vas deferens, a forme fruste of the $\mathrm{CF}$ disease, a normal baseline nasal transepithelial potential difference (PD) but an impaired response to pharmacological interventions have been reported. The purpose of the present study was to explore ion transport in respiratory epithelium from patients with disseminated bronchiectasis.

The PD under both baseline and pharmacological interventions was investigated in 13 healthy subjects, six patients with genetically proven $C F$ and 15 patients with disseminated bronchiectasis as confirmed by computed tomography scan.

Baseline PD was similar in the control and bronchiectasis groups but, as expected, was significantly more negative in the $C F$ group. Patients with bronchiectasis responded to pharmacological tests (sequential perfusion with amiloride, chloride-free solution, isoprenaline and uridine triphosphate (UTP) similarly to healthy subjects. In contrast, $C F$ patients exhibited an increased response to amiloride and an impaired response to chloride-free solution and isoprenaline.

The data show that patients with disseminated bronchiectasis exhibit normal electrophysiological properties in their nasal epithelium. Nasal transepithelial potential difference including pharmacological tests may appear a valuable diagnostic procedure for cystic fibrosis with disseminated bronchiectasis.

Eur Respir J 1999; 13: 1276-1280.

Cystic fibrosis (CF), the most frequent lethal autosomal recessive disorder in Caucasians, is characterized by defective electrolyte transport across secreting epithelia [13]. The CF gene product, cystic fibrosis transmembrane conductance regulator (CFTR), is a plasma membrane chloride channel that also acts as a regulator of heterologous ionic channels [4-6]. The CF phenotype includes disseminated bronchiectasis, pancreatic insufficiency, meconium ileus, congenital bilateral absence of the vas deferens (CBAVD), and elevated sweat chloride concentration.

Disseminated bronchiectasis is another pathological entity, first described by Laënnec in 1819 [7], which is characterized by an abnormal and permanent dilatation of the bronchial tree. Principal aetiological factors for diffuse bronchiectasis include infectious injuries during childhood, primary ciliary dyskinesia, and mucus disorders. However, the nosological limit between CF and disseminated bronchiectasis remains vague as: 1) classical CF has long been recognized as a cause of disseminated bronchiectasis [7]; 2) an increased incidence of heterozygous $\Delta \mathrm{F} 508$ mutations in the $\mathrm{CF}$ gene has been repetitively reported in patients with bronchiectasis [811]; and 3) disseminated bronchiectasis may represent the unique clinical symptom in mild forms of genetically proven $C F$ in adult patients $[12,13]$. Similarly, the limits between isolated CBAVD and CF are also subject to discussion as: 1) patients with CBAVD have an abnor-
*Laboratoire des Explorations Fonctionnelles and Service de Pneumologie, Hôpital G \& R Laënnec; ${ }^{+}$INSERM CJF 96.01, and **Laboratoire de Génétique Moléculaire, Hôpital Hôtel-Dieu, 44093 Nantes, France.

Correspondence: D. Escande

INSERM CJF 96.01

Hôpital Hôtel-Dieu

BP 1005

44093 Nantes

France

Fax: 33240412950

Keywords: Bronchiectasis cystic fibrosis nasal potential difference

Received: January 191998

Accepted after revision December 71998

This work was supported by grants from the Association Française de Lutte contre la Mucoviscidose (AFLM). mally high incidence of heterozygous mutations in the CF gene [14]; and 2) CBAVD may represent the unique clinical manifestation of a mild CF disease [15].

Measurement of sweat chloride is a classical test to diagnose $\mathrm{CF}$ among patients with disseminated bronchiectasis or CBAVD, although this technique occasionally provides borderline values $[16,17]$. Measurement of baseline nasal transepithelial potential difference (nasal PD) is another test to diagnose CF $[18,19]$. Patients with disseminated bronchiectasis or isolated CBAVD have normal basal nasal PD $[20,21]$, whereas patients with $C F$ exhibit nasal PD hyperpolarization. However, dynamic $\mathrm{PD}$ recordings using pharmacological tests performed in patients with CBAVD have demonstrated a defective chloride conductance, but a normal sodium conductance [21]. It was therefore decided to explore whether nasal PD recordings in a cohort of adult patients with disseminated bronchiectasis would demonstrate dynamic anomalies unrevealed by baseline PD measurements.

\section{Materials and methods}

\section{Potential difference measurement}

Nasal PD was measured using a method described previously by KNOWLES et al. [18] and modified by ALTON et al. [22]. The reference electrode consisted of a calomel 
Table 1. - Clinical data, cystic fibrosis transmembrane conductance regulator (CFTR) genotypes and sweat chloride concentrations in cystic fibrosis patients

\begin{tabular}{|c|c|c|c|c|c|}
\hline $\begin{array}{l}\text { Patient } \\
\text { No. }\end{array}$ & $\begin{array}{c}\text { Age } \\
\text { yrs }\end{array}$ & Mutations & $\begin{array}{c}\text { Sweat chloride } \\
\mathrm{mEq} \cdot \mathrm{L}^{-1}\end{array}$ & $\mathrm{PI} / \mathrm{PS}$ & $\begin{array}{c}\text { Heart-Lung } \\
\text { Transplantation }\end{array}$ \\
\hline 1 & 22 & $\Delta \mathrm{F} 508 / \Delta \mathrm{F} 508$ & 88 & PI & No \\
\hline 2 & 21 & $\Delta \mathrm{F} 508 / 3659 \mathrm{delC}$ & NA & PI & Yes \\
\hline 3 & 26 & $\Delta \mathrm{F} 508 / \Delta \mathrm{F} 508$ & NA & PI & Yes \\
\hline 4 & 24 & $\Delta \mathrm{F} 508 / \Delta \mathrm{F} 508$ & 190 & PI & No \\
\hline 5 & 28 & $\Delta \mathrm{F} 508 / \Delta \mathrm{F} 508$ & NA & PI & No \\
\hline 6 & 23 & $\Delta \mathrm{F} 508 / \Delta \mathrm{F} 508$ & NA & PI & Yes \\
\hline 7 & 61 & $\Delta \mathrm{F} 508 / \mathrm{L} 206 \mathrm{~W}$ & 100 & PS & No \\
\hline
\end{tabular}

PI: pancreatic insufficiency; PS: pancreatic sufficiency; NA: not available.

electrode (K401 Radiometer; Radiometer Analytical SA, Villeurbanne, France) connected to a $20-\mathrm{G}$ needle filled with $2.5 \%$ agar and placed subcutaneously in the forearm. The exploring electrode also consisted of a calomel electrode connected to a size 12 Foley (Laboratoire BARD SA, Trappes, France) catheter placed in the nasal cavity and perfused with a control solution containing $(\mathrm{mM}): \mathrm{Na}^{+} 140, \mathrm{~K}^{+} 6, \mathrm{Mg}^{2+} 1, \mathrm{Ca}^{2+} 2, \mathrm{Cl}^{-} 152$, and glucose 10 , titrated to $\mathrm{pH} 7.4$ at room temperature with Hepes ( $N$-(2-hydroxyethyl)-piperazine- $N$-(2-ethane-sulphonic-acid)). Both electrodes were connected to a high impedance $\left(10^{11} \Omega\right.$ ) voltmeter (A-M Systems; Phymep, Paris, France). Prior to use, the two electrodes were placed in close contact to set the $0 \mathrm{mV}$ level. The Foley catheter was placed along the floor of the nasal cavity and fixed at the most negative stable PD. In order to evaluate the response of nasal PD to perfusion with pharmacological agents, parallel perfusion lines were placed and perfused sequentially. Solution 1 was the control solution supplemented with amiloride $10^{-4} \mathrm{M}$. Solution 2 was a chloride-free solution with gluconate as a substitute, also containing amiloride $10^{-4} \mathrm{M}$. Solution 3 was solution 2 supplemented with isoprenaline $10^{-5} \mathrm{M}$. Solution 4 was solution 3 supplemented with uridine triphosphate (UTP) $10^{-4} \mathrm{M}$. Perfusion with the chloride-free solution induced a junctional potential that was not compensated for.

\section{Genotypic analysis}

Mutations of CFTR were screened by the amplification refractory mutation system (ARMS). This method allowed the detection of seven common mutations: $621+1 \mathrm{G} \rightarrow \mathrm{T}$, $\Delta \mathrm{F} 508, \mathrm{G} 542 \mathrm{X}, \mathrm{N} 1303 \mathrm{~K}, \mathrm{G} 551 \mathrm{D}, 1717-1 \mathrm{G} \rightarrow \mathrm{A}$, and 3659delC [23].

\section{Subjects}

Healthy subjects. Thirteen normal nonsmoking subjects (six females and seven males, age range 23-52 yrs, median age $32 \mathrm{yrs}$ ) were included in the study. They had no history of respiratory disease. None were taking any medication other than an oral contraceptive.

Patients. Six CF patients (three females and three males, age range 21-28 yrs, median age 24 yrs) were included in this study. Clinical criteria, sweat chloride concentrations and mutations are summarized in table 1 . The six $\mathrm{CF}$ patients exhibited a typical clinical CF phenotype confirmed by genotypic analysis. Three CF patients had heart-lung transplants. A previous work demon- strated that $\mathrm{CF}$ patients with or without heart-lung transplant show no difference in their nasal PD [24]. An additional patient with bronchiectasis (patient No. 7) was included in the CF group for statistical analysis on the basis that the nasal PD measurement under pharmacological challenge was suggestive for $\mathrm{CF}$, a diagnosis confirmed by genotypic analysis.

Fifteen patients with diffuse bronchiectasis proved on CT scan (11 females and four males, age range 17-62 yrs, median age 41 yrs) were also included. Their clinical profile is summarized in table 2. Genotypic analysis of CFTR was conducted in 12/15 patients with disseminated bronchiectasis. Among these, 11 showed no mutation in the CF gene.

\section{Statistical analysis}

All data are presented as mean \pm SEM and have been tested for normality by the Kolmogorov-Smirnov test. Depending on the outcome of the test, parametric or nonparametric one-way analysis of variance (ANOVA) was performed with repeated measures and non-repeated measures for data. When an overall significant difference was found, pair-wise comparisons were performed with the group of patients with bronchiectasis as the reference group. The Dunn correction for non-normally distributed data was used for $10^{-5} \mathrm{M}$ isoprenaline and $10^{-4} \mathrm{M} \mathrm{UTP}$, and Dunnett correction for normally distributed data for chloride-free

Table 2. - Clinical data and sweat chloride concentrations in patients with disseminated bronchiectasis

\begin{tabular}{|c|c|c|c|c|c|}
\hline \multirow{2}{*}{$\begin{array}{l}\text { Patient } \\
\text { No }\end{array}$} & \multirow{2}{*}{$\begin{array}{l}\text { Age } \\
\text { yrs }\end{array}$} & \multirow{2}{*}{$\begin{array}{c}\text { Sweat Chloride } \\
\mathrm{mEq} \cdot \mathrm{L}^{-1}\end{array}$} & \multicolumn{2}{|c|}{ FEV1 } & \multirow{2}{*}{$\begin{array}{c}\text { Pseudomonas } \\
\text { aeruginosa }\end{array}$} \\
\hline & & & $\mathrm{L}$ & $\%$ pred & \\
\hline 7 & 61 & 100 & 0.70 & 28 & No \\
\hline 8 & 52 & 24 & 1.5 & 40 & Yes \\
\hline 9 & 42 & 48 & 0.93 & 40 & Yes \\
\hline 10 & 49 & NA & 1.26 & 46 & Yes \\
\hline 11 & 30 & NA & 4.71 & 115 & No \\
\hline 12 & 26 & 14 & 2.12 & 63 & No \\
\hline 13 & 62 & 12 & 0.54 & 21 & Yes \\
\hline 14 & 48 & NA & 1.20 & 43 & Yes \\
\hline 15 & 48 & NA & \multicolumn{2}{|c|}{ NA } & No \\
\hline 16 & 32 & 12 & 0.79 & 25 & Yes \\
\hline 17 & 25 & 9 & 2.01 & 64 & No \\
\hline 18 & 51 & NA & 0.64 & 21 & No \\
\hline 19 & 43 & NA & 1.64 & 49 & No \\
\hline 20 & 42 & NA & 2.40 & 54 & No \\
\hline 21 & 17 & 50 & 2.97 & 90 & No \\
\hline
\end{tabular}

FEV1: forced expiratory volume in one second; NA: not available. Patient 7 was also included in the cystic fibrosis group. 


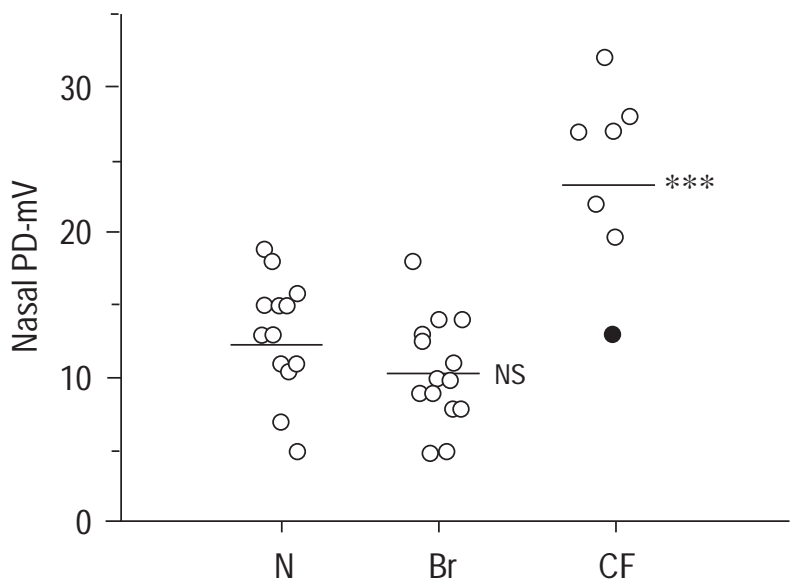

Fig. 1. - Baseline potential difference (PD) measured in 13 normal subjects $(\mathrm{N}), 14$ patients with bronchiectasis $(\mathrm{Br})$, and seven patients with cystic fibrosis $(\mathrm{CF})$. The horizontal bar for each group represents mean PD. : patient no. 7. ***: $\mathrm{p}<0.001$ versus normal subjects.

solution containing amorilide. The Student-NewmanKeuls correction was used for pair-wise comparisons involving a given experimental condition to the previous one. For ANOVA and pair-wise comparisons, the significance level was set at $\mathrm{p}<0.05$. All statistical analyses were performed with Sigmastat version 2.0.3 (SPSS, Germany) software.

\section{Results}

\section{Basal nasal potential difference}

Basal PD was determined in the control Hepes-buffer solution. The averaged transepithelial PD in patients with bronchiectasis was not significantly different from that recorded in healthy control subjects (fig. 1). In contrast, the basal PD in patients with CF was about two-fold more negative than in healthy volunteers or in patients with bronchiectasis. There was some degree of overlap in individual values between patients with $\mathrm{CF}$ and patients with bronchiectasis or healthy volunteers.

\section{Nasal response to amiloride}

Amiloride, a specific sodium channel blocker [25, 26], was applied locally at a final concentration of $10^{-4} \mathrm{M}$. In the three groups, the nasal PD became significantly less negative under the effects of amiloride (fig. 2). In the CF group, the decrease in nasal PD was about two-fold more pronounced than in either the healthy group or the bronchiectasis group (fig. 3). In contrast, there was no statistical difference in the response to amiloride between the control and bronchiectasis groups.

\section{Nasal response to chloride-free solution}

The nasal epithelium was then superfused with a chloride-free extracellular medium (solution 2). The amiloride concentration was maintained constant throughout in the superfusate so that the changes induced by chloride substitution only reflected an increase in the driving force for chloride ions. Normal subjects and bronchiectasis patients exhibited a moderate hyperpolarization of their nasal PD in response to chloride-free superfusion (fig. 2). These two groups did not differ significantly with respect to their responses to chloride-free superfusion (fig. 3). The nasal PD in patients with CF was unmodified by superfusion with chloride-free solution (figs. 2 and 3 ). In this respect, the difference between bronchiectasis and CF subjects reached significance (fig. 3 ).
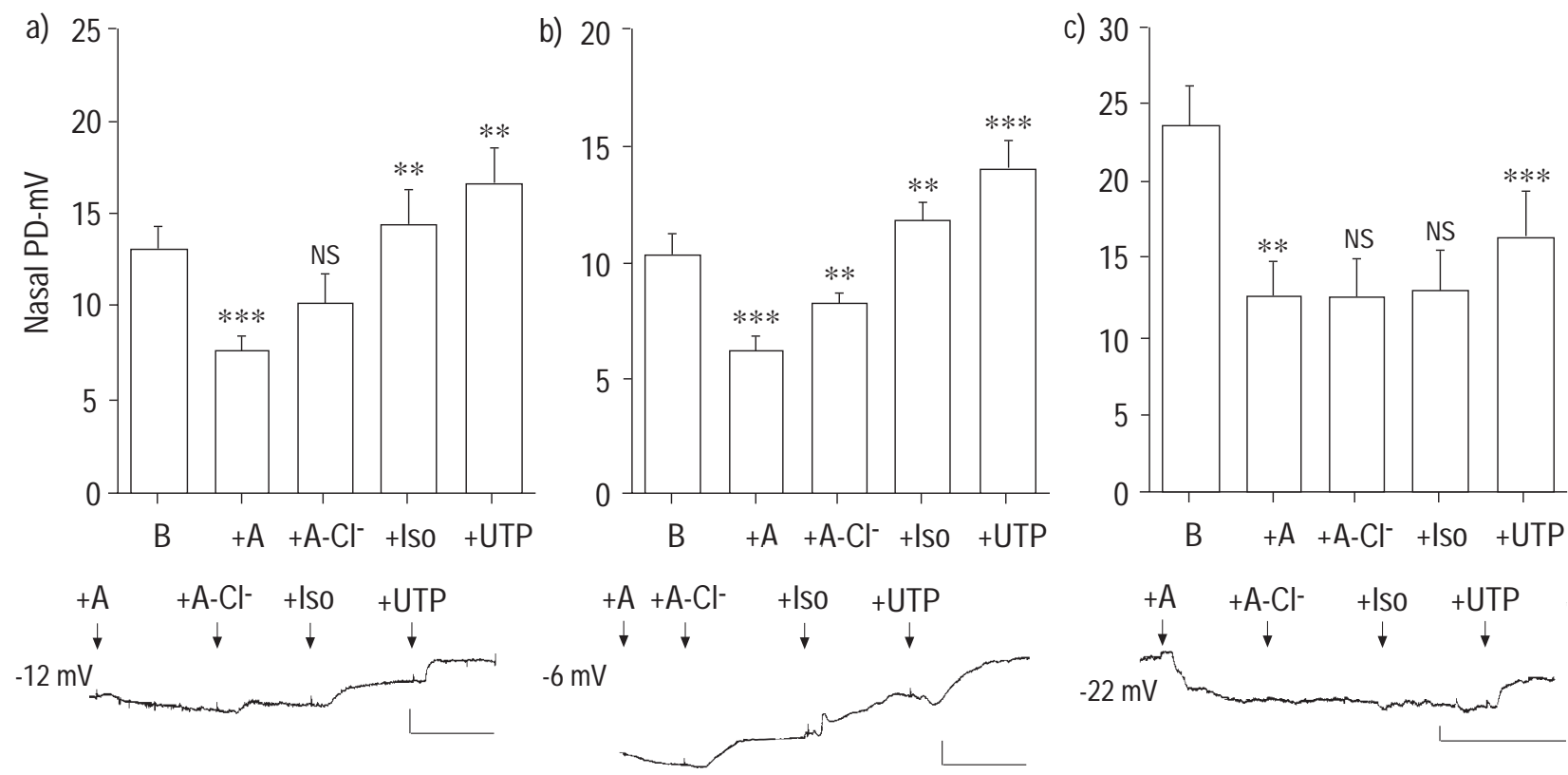

Fig. 2. - Nasal potential difference (PD) during pharmacological tests. a) healthy subjects $(n=13)$; b) bronchiectasis patients ( $=14)$, and c) cystic fibrosis patients $(\mathrm{n}=7)$. The top panel shows averaged data and the lower panel shows a typical nasal PD recording. Nasal epithelium surface was superfused in sequence with control solution (basal (B)); amiloride $10^{-4} \mathrm{M}(+\mathrm{A})$, chloride-free solution containing amiloride $\left(+\mathrm{A}-\mathrm{Cl}^{-}\right)$; isoprenaline $10^{-5} \mathrm{M}(+\mathrm{Iso})$ and uridine triphosphate $10^{-4} \mathrm{M}(+\mathrm{UTP})$. Statistical analysis conducted in comparison with nasal PD values before each pharmacological intervention. $* * *$ : $\mathrm{p}<0.001 ; * *: \mathrm{p}<0.01 ; *$ : $\mathrm{p}<0.05$ versus basal. 


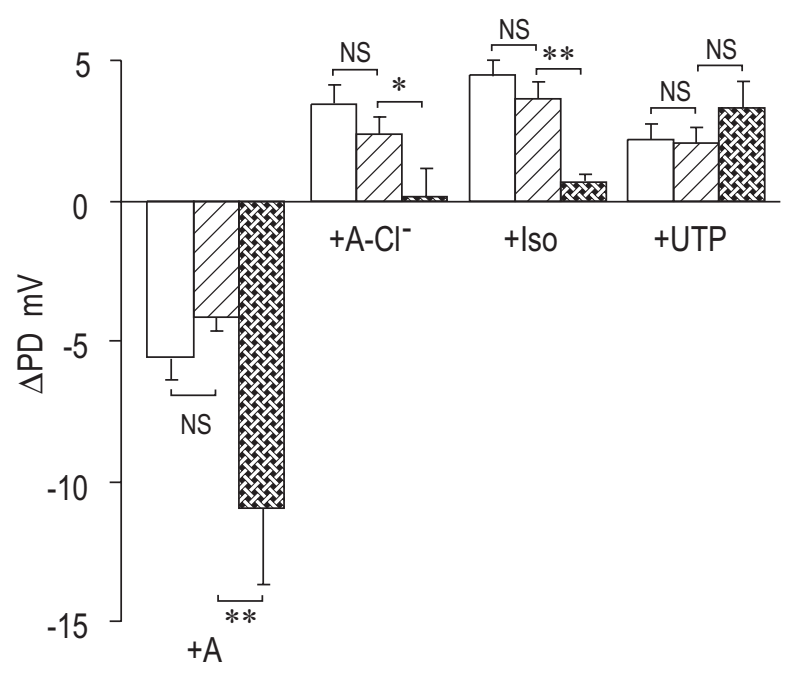

Fig. 3. - Comparison between groups for the nasal potential difference $(\mathrm{PD})$ response to perfusion with amiloride $(+\mathrm{A})$, chloride-free solution ( $+\mathrm{A}^{-} \mathrm{Cl}^{-}$), isoprenaline (+Iso), and uridine triphosphate (UTP). $\square$ : Healthy subjects; $\mathbb{Z}$ : patients with bronchiectasis; : patients with cystic fibrosis. Data are mean \pm SEM of the nasal PD change. Statistical analysis conducted in comparison with values in the healthy subject group. **: $\mathrm{p}<0.01 ; *$ : $<<0.05$.

\section{Nasal response to isoprenaline}

Isoprenaline, a $\beta$-receptor agonist that raises intracellular cyclic adenosine monophosphate (cAMP) and thereafter activates CFTR, was perfused at a final concentration of $10^{-5} \mathrm{M}$ in the chloride-free solution containing amiloride (solution 3). In normal subjects and patients with bronchiectasis, the nasal PD became more negative under the effects of isoprenaline with no statistical difference between the two groups (fig. 3). In contrast, the nasal PD of $\mathrm{CF}$ patients did not hyperpolarize in response to isoprenaline. The difference between normal subjects or bronchiectasis patients and CF patients was highly significant (fig. 3).

\section{Nasal response to uridine triphosphate}

Finally, UTP was added to the precedent superfusate and applied locally to the nasal epithelium surface. UTP supposedly triggers chloride secretion through stimulation of an extracellular P2U receptor coupled to an outward rectifying chloride channel [6]. As shown in figures 2 and 3 , UTP induced hyperpolarization of the nasal PD in control subjects, CF patients and bronchiectasis subjects. Hyperpolarization as produced by UTP in the CF population was not significantly different in amplitude from that observed in the normal or in the bronchiectasis populations.

\section{Patient No. 7 case report}

Patient No. 7 was a female aged 61 yrs who was treated for many years for disseminated bronchiectasis. Diagnosis was confirmed on chest radiograph and computed tomography scan. Sputum culture was positive for Staphylococcus aureus. Pulmonary function was altered with a forced expiratory volume in one second $(\mathrm{FEV} 1)$ of $0.70 \mathrm{~L}$ (28\% predicted) and a forced vital capacity (FVC) of 1.58 L ( $57 \%$ pred). There was no family history of CF. She had no fertility problems and presented no digestive symptoms. The diagnosis of CF was suspected because of repeated positive sweat chloride tests $(100 \mathrm{mM})$. Her basal nasal PD was within normal limits $(-13 \mathrm{mV}$; fig. 1$)$. The response of the nasal PD to amiloride superfusion gave a voltage depolarization of $5 \mathrm{mV}$, i.e. within the normal range. Nasal PD did not change further under superfusion with either chloride-free solution or isoprenaline. Finally, UTP stimulation hyperpolarized the nasal $\mathrm{PD}$ to $-17 \mathrm{mV}$. Despite her baseline PD value being close to normal, the lack of hyperpolarization produced by chloride-free solution or isoprenaline prompted the screening for mutations in her CF gene. This revealed $\Delta$ F508/L206W CFTR gene mutations.

\section{Discussion}

The present work demonstrates that nasal PD both under baseline and pharmacological interventions in patients with bronchiectasis is indistinguishable from that measured in a normal healthy population, indicating that the bioelectrical properties of nasal epithelia, including both the sodium and chloride transport processes, are normal in patients with bronchiectasis. AlTON et al. [20] have previously demonstrated that the baseline PD in patients with bronchiectasis and in patients with Young's syndrome is normal. Data from the present study are in line with this observation. In addition, nasal PD under pharmacological interventions is normal in patients with bronchiectasis. This is an important finding as baseline PD in CBAVD patients is normal but the response to pharmacological agents is altered.

The incidence of CF among adult patients with disseminated bronchiectasis is unknown. A prospective work investigated 38 patients with bronchiectasis with the aim of identifying possible aetiological factors using ultrastructural and functional studies of respiratory cilia, sweat test, semen analysis, and concentration of $\alpha_{1}$-protease inhibitor and immunoglobulin [27]. The diagnosis of CF was suspected in 6/38 subjects but was not confirmed by genotype analysis. Another study by Dumur et al. [8] examined 65 adults with bronchorrhoea. In one of their patients, genotype analysis revealed a forme fruste of CF (sweat chloride $100 \mathrm{mmol} \cdot \mathrm{L}^{-1}$ ). Five other patients were heterozygote carriers for the $\Delta \mathrm{F} 508$ mutation. This corresponds to an incidence of heterozygote carriers of $1 / 13$, differing from the expected incidence of $1 / 25$ in the general population. Another study reported five different CFTR gene mutations in a series of 16 patients with diffuse bronchiectasis [10]. Finally, GIRODON et al. [11] identified 11 patients carrying CFTR mutations in a cohort of 32 patients with bronchiectasis. Taken together, these studies strongly suggest a link between CFTR mutation and bronchiectasis through an as yet unidentified mechanism.

In the case of patient No. 7 in the present study, the baseline nasal PD was within normal limits. However, the absence of significant change in the PD with isoprenaline stimulation was highly suggestive for $\mathrm{CF}$, a diagnosis confirmed by genotype analysis which revealed $\Delta$ F508/ 
L206W mutations. The L206W mutation, located in exon $6 \mathrm{a}$, is usually associated with a mild CF phenotype (bronchiectasis or CBAVD alone) or even asymptomatic presentations of CF $[12,13]$.

The most significant consequence of this study is that nasal transepithelial potential difference including pharmacological tests may be a valuable and rapid test to diagnose cystic fibrosis patients with predominant bronchiectasis manifestations. Further prospective studies are needed to evaluate the reliability of this test in this context.

Acknowledgements. The authors thank S. Baudet for expert assistance with statistical analysis.

\section{References}

1. Quinton PM. Cystic fibrosis: a disease in electrolyte transport. FASEB J 1990; 4: 2709-2717.

2. Widdicombe JH, Kondo M, Mochizuki H. Regulation of airway mucosal ion transport. Int Arch Allergy Appl Immunol 1991; 94: 56-61.

3. Liedtke CM. Electrolyte transport in the epithelium of pulmonary segments of normal and cystic fibrosis lung. FASEB J 1992; 6: 3076-3084.

4. Riordan JR, Rommens JM, Kerem BS, et al. Identification of the cystic fibrosis gene: cloning and characterization of complementary DNA. Science 1989; 245: 1066-1073.

5. Anderson MP, Gregory RJ, Thompson S, et al. Demonstration that CFTR is a chloride channel by alteration of its anion selectivity. Science 1991; 253: 202-207.

6. Gabriel SE, Carke LL, Boucher RC, Stutts MJ. CFTR and outward rectifying chloride channels are distinct proteins with a regulatory relationship. Nature 1993; 363: 263266.

7. Barker AF, Bardana EJ. Bronchiectasis: update of an orphan disease. Am Rev Respir Dis 1988; 137: 969-978.

8. Dumur V, Lafitte JJ, Gervais R, et al. Abnormal distribution of cystic fibrosis $\Delta \mathrm{F} 508$ allele in adults with chronic bronchial hypersecretion. Lancet 1990; 336: 1340.

9. Gervais R, Lafitte JJ, Dumur V, et al. Sweat chloride and $\Delta$ F508 mutation in chronic bronchitis or bronchectasis. Lancet 1993; 342: 997.

10. Pignatti PF, Bombieri C, Marigo C, Benetazzo M, Luisetti $\mathrm{M}$. Increased incidence of cystic fibrosis gene mutations in adults with disseminated bronchiectasis. Hum Mol Genet 1995: 4: 635-639.

11. Girodon E, Cazeneuve C, Lebargy F, et al. CFTR gene mutations in adults with disseminated bronchiectasis. Eur J Hum Genet 1997; 5: 149-155.

12. Rozen R, Ferreira-Rajabi L, Robb L, Colman N. L206W mutation of the cystic fibrosis gene, relatively frequent in French Canadians, is associated with atypical presentations of cystic fibrosis. Am J Med Genet 1995; 57: 437439.
13. Desgeorges M, Rodier M, Piot M, Demaille J, Claustres M. Four adult patients with the missense mutation L206W and a mild cystic fibrosis phenotype. Hum Genet 1995; 96: 717-720.

14. Dumur V, Gervais R, Rigot JM, et al. Abnormal distribution of CF $\Delta \mathrm{F} 508$ allele in azoospermic men with congenital aplasia of epididymis and vas deferens. Lancet 1990; 336: 512.

15. Anguiano A, Oates RD, Amos JA, et al. Congenital bilateral absence of the vas deferens: a primarily genital form of cystic fibrosis. JAMA 1992; 267: 1794-1797.

16. Highsmith WE, Burch LH, Zhou Z, et al. A novel mutation in the cystic fibrosis gene in patients with pulmonary disease but normal sweat chloride concentrations. N Engl J Med 1994; 331: 974-980.

17. Stewart B, Zabner J, Shuber AP, Welsh MJ, McCray PB. Normal sweat chloride values do not exclude the diagnosis of cystic fibrosis. Am J Respir Crit Care Med 1995; 151: 899-903.

18. Knowles MR, Carson JL, Collier AM, Gatzy JT, Boucher RC. Measurements of nasal transepithelial electric potential differences in normal human subjects in vivo. Am Rev Respir Dis 1981; 124: 484-490.

19. Knowles MR, Gatzy JT, Boucher RC. Increased bioelectric potential difference across respiratory epithelia in cystic fibrosis. N Engl J Med 1981; 305: 1489-1495.

20. Alton EWFW, Hay JG, Munro C, Geddes DM. Measurement of nasal potential difference in adult cystic fibrosis, Young's syndrome, and bronchiectasis. Thorax 1987; 42: 815-817.

21. Osborne LR, Lynch M, Middleton PG, et al. Nasal epithelial ion transport and genetic analysis of infertile men with congenital bilateral absence of the vas deferens. Hum Mol Genet 1993; 2: 1605-1609.

22. Alton EWFW, Currie D, Logan-Sinclair R, Warner JO, Hodson ME, Geddes DM. Nasal potential difference: a clinical diagnostic test for cystic fibrosis. Eur Respir $J$ 1990; 3: 922-926.

23. Ferrie RM, Schwarz MJ, Robertson NH, et al. Development, multiplexing, and application of ARMS tests for common mutations in the CFTR gene. Am J Hum Genet 1992; 51: 251-262.

24. Wood A, Higenbottam T, Jackson M, Scott J, Stewart S, Wallwork J. Airway mucosal bioelectric potential difference in cystic fibrosis after lung transplantation. $\mathrm{Am}$ Rev Respir Dis 1989; 140: 1645-1649.

25. Middleton PG, Geddes DM, Alton EWFW. Protocols for in vivo measurement of the ion transport defects in cystic fibrosis nasal epithelium. Eur Respir J 1994; 7: 20502056.

26. Knowles MR, Paradiso AM, Boucher RC. In vivo nasal potential difference: techniques and protocols for assessing efficacy of gene transfer in cystic fibrosis. Hum Gene Ther 1995; 6: 445-455.

27. Verra F, Escudier E, Bignon J, et al. Inherited factors in diffuse bronchiectasis in the adult: a prospective study. Eur Respir J 1991; 4: 937-944. 